\title{
DEBATES
}

\section{Partidos en el gobierno: activismo político, centralización interna y subordinación al liderazgo gubernamental}

\section{Parties in government: political activism, internal centralization and subordination to government leadership}

\section{Rosa María Marcuzzi}

\section{Resumen}

El presente estudio analiza el cambio de la organización interna de los partidos gubernamentales. Siguiendo la orientación teórica propuesta por el politólogo noruego Kaare Strom sobre el comportamiento de los partidos políticos en las democracias contemporáneas, el análisis compara el Partido de los Trabajadores de Brasil, el Frente para la Victoria de Argentina, el Frente Amplio de Uruguay y el Movimiento Alianza Patria Altiva I Soberana de Ecuador en sus primeros períodos de gobierno. El objetivo del estudio es analizar los cambios organizativos de los partidos en el ejercicio del gobierno. El estudio concluye que los partidos en el ejercicio de gobierno presentan una combinación de creciente activismo político, centralización interna y subordinación al liderazgo gubernamental, lo que contradice la teoría desarrollada por Strom.

\section{Palabras clave}

Partidos Gubernamentales; Activismo; Centralización Interna; Liderazgo Gubernamental.

\section{Abstract}

This study analyses the change in the internal organization of government parties. Following the theoretical orientation proposed by Norwegian political scientist Kaare Strom on the behavior of political parties in contemporary democracies, the analysis compares the Brazilian Workers' Party, the Front for Victory of Argentina, the Broad Front of Uruguay and Ecuador's Proud and Sovereign Homeland Alliance Movement in their early periods of government. The objective of the study is to analyze the organizational changes of parties in the exercise of government. The study concludes that parties in the exercise of government present a combination of growing political activism, internal centralization and subordination to government leadership, contradicting the theory developed by Strom.

\section{Keywords}

Government Parties; Activism; Internal Centralization; Government Leadership. 


\section{4 | Rosa María Marcuzzi}

\section{Introducción}

Los partidos políticos se distinguen de las demás organizaciones sociales por monopolizar la representación política, disposición incorporada en los ordenamientos legales de las democracias contemporáneas. Los partidos son actores sociales y estatales simultáneamente, interrelacionan entre la sociedad y los poderes políticos estatales, doble presencia que condiciona la evolución de su organización interna.

La organización interna de los partidos políticos ha sido objeto de los estudios clásicos de la Politología, desde el análisis de Robert Michels a inicios del siglo XX, pasando por los estudios de Duverger, Kirchheimer, Panebianco y Strom en el transcurso del siglo XX, llegando en el presente siglo a las investigaciones de Katz y Mair, Ware, Wolinetz y Samuels y Shugart (AMARAL, 2013).

Desde la perspectiva del institucionalismo racional, el influyente trabajo de Kaare Strom (1990) sobre el comportamiento de los partidos políticos en las democracias contemporáneas afirma que las organizaciones partidarias que privilegian el activismo de sus miembros, descentralizan la toma de decisiones, de tal manera que los miembros del partido, en sus diversos niveles, se comprometan en las actividades y en los cursos de acción política adoptados, y el partido adquiere autonomía organizacional en relación a los miembros del partido que desempeñan cargos públicos.

La evidencia empírica de partidos gubernamentales latinoamericanos contradice la afirmación sostenida por el politólogo noruego. El Partido de los Trabajadores de Brasil, el Frente para la Victoria de Argentina, el Frente Amplio de Uruguay y el Movimiento Alianza Patria Altiva I Soberana de Ecuador, en sus primeros períodos de gobierno, presentaron un creciente activismo político que se correspondió con una progresiva centralización interna y con la subordinación al liderazgo gubernamental. En los partidos citados, el activismo partidario se incrementó de manera simultánea a la centralización en la toma de decisiones al interior de la organización y a la creciente subordinación al liderazgo gubernamental.

El presente estudio compara la combinación de activismo partidario, centralización interna y subordinación al liderazgo gubernamental presente en el Partido de los Trabajadores de Brasil, el Frente para la Victoria de Argentina, el Frente Amplio de Uruguay y el Movimiento Alianza Patria Altiva I Soberana de Ecuador en sus primeros períodos de gobierno. El propósito del estudio es contribuir con investigaciones empíricas al conocimiento sobre el cambio de los partidos gubernamentales latinoamericanos. 
El análisis se desarrolla en siete apartados. En el primer apartado se enuncia el enfoque teórico, la metodología empleada y los casos seleccionados, en los siguientes cuatro apartados se analizan los casos seleccionados, en el sexto apartado se compara a los partidos gubernamentales, para concluir con unas breves reflexiones.

\section{E1 Partido de los Trabajadores de Brasil: activismo politico, centralización interna $y$ subordinación al liderazgo gubernamental (2003-2007)}

El Partido de los Trabajadores de Brasil (en adelante PT) surgió representando a una diversidad de posiciones políticas de la izquierda brasilera, a ex militantes comunistas críticos de los socialismos reales, al nuevo sindicalismo, a sectores católicos progresistas provenientes de la Teología de la Liberación de las Comunidades Eclesiales de Base y a ex legisladores del Partido del Movimiento Democrático Brasilero (PEREIRA DA SILVA, 2011). El reconocimiento como partido político lo obtuvo el 10 de febrero de 1980, al otorgarle la personería jurídica por el Tribunal Superior de Justicia Electoral.

Su organización fue casi simultánea con la creación de la Central Única de Trabajadores - CUT, ambas organizaciones contaban con antecedentes en las luchas políticas y sindicales, en las huelgas de la industria automovilística de 1978, que enfrentaron al movimiento obrero con la patronal y la dictadura militar (MARINI, 1986). El partido político y el sindicato compartieron el liderazgo de Luis Inácio Lula da Silva, obrero metalúrgico, quien fue simultáneamente presidente del partido y presidente del Sindicato de Metalúrgicos del ABC Paulista, sindicato urbano miembro fundador de la CUT. Las autoridades de la CUT formaron parte de la Comisión Ejecutiva de la Dirección Nacional del partido.

Desde su origen el partido y la Central de Trabajadores se vincularon con los movimientos sociales. La CUT, desde la Primera Conferencia Nacional de la Clase Trabajadora de 1981, impulsó la reforma agraria, demanda que tendrá en el Movimiento de Trabajadores Rurales Sin Tierra - MST su profundización. El MST se creó en 1984 y desde esa fecha organizó encuentros anuales y congresos a través de los cuales se constituyó la Confederación de Cooperativas de Reforma Agraria de Brasil y el Instituto Técnico de Capacitación e Investigación de la Reforma Agraria. La mayoría de los miembros del MST pertenecen al PT (MIRZA, 2006). Su Congreso Nacional celebrado en el año 2003 contó con la participación de Lula, ya

\footnotetext{
${ }^{1}$ Tales fueron los casos de Ricardo Berzoini, Delubio Soares, Olívio Dutra y Luiz Marinho.
} 
siendo Presidente de Brasil (MIRZA, 2006). El PT promovió el Foro Social Mundial - FSM y financió la organización del Foro en la ciudad de Porto Alegre antes de acceder a la Presidencia de la Nación (BRECKENRIDGE-JACKSON et al., 2017).

Durante la década del 90, el PT abandonó la expresión socialismo y moderó su programa partidario, proceso que se expresó en la Carta al Pueblo Brasilero de junio de 2002, donde se explicitaba el estrecho margen de maniobra de la política económica de un futuro gobierno, la necesidad de mantener el equilibrio fiscal, el superávit primario y controlar el gasto público (SECCO, 2018). Este proceso de moderación del programa partidario del PT no estuvo exento de conflictos internos, la Carta al Pueblo Brasilero fue llamada "Carta a los Banqueros" por las tendencias de izquierda internas del partido (SECCO, 2018).

El estatuto partidario del PT regula la participación de los movimientos sociales desde los núcleos de base hacia todos los niveles de la organización, municipal, estadual y nacional, en las llamadas "sectoriales" de la juventud, de género, agraria y medio ambiente. Los secretarios de las sectoriales integran, con derecho a voz, las direcciones del partido en todos sus niveles. Los presidentes del partido de todos los niveles, municipal, zonal, estadual y nacional son electos de forma separada, con ballotage si no ha alcanzado la mayoría absoluta de votos en la primera vuelta (PT, 2007 y 2001).

La participación interna de los afiliados se regula a través del proceso de elección directa de todas las autoridades partidarias. Los afiliados que cuenten con más de un año de afiliación pueden postularse a cargos partidarios y participar en la elección de todos los organismos de dirección política del partido mediante el proceso de elección directa (PED) en todos los niveles de actuación, municipal, zonal, estatal y nacional. Los afiliados pueden organizar tendencias al interior del partido para manifestar sus posiciones políticas (PT, 2007 y 2001).

El sistema electoral utilizado en las elecciones internas es proporcional, integrando a las minorías y con un cupo femenino del 30 por ciento. Las elecciones internas del partido se celebran cada tres años (PT, 2007 y 2001).

En septiembre de 2003, la Dirección Nacional del partido lanzó una campaña de afiliación para incrementar la presencia partidaria en los gobiernos locales. El número de afiliados se incrementó desde 200.000 a 800.000 afiliados en el período 2001-2005 (VAN DYCK, 2014)². El partido se expandió desde la Dirección

\footnotetext{
${ }^{2}$ El número de afiliados sumó casi 1.400 .000 al concluir el segundo gobierno de Lula en el año 2010 (SECCO, 2018).
} 
Nacional hacia lo niveles subnacionales, respondiendo a una estrategia de expansión territorial para incrementar el voto local, especialmente en los municipios del Nordeste, donde no contaba con una significativa adhesión electoral (VAN DYCK, 2014).

La elección interna del PT, como partido gubernamental, fue convocada de forma anticipada por la Dirección Nacional el 18 de septiembre de $2005^{3}$. La convocatoria anticipada se debió a la renuncia de sus titulares por escándalos de corrupción ${ }^{4}$. En las elecciones internas participaron siete listas, la lista oficialista "Campo Mayoritario" y seis listas de la oposición. De los 840.108 afiliados, participaron 312.000 electores. Ricardo Berzoini, candidato a la presidencia por la lista oficial, no logró la mayoría en primera vuelta. Berzoini contó con el 42 por ciento de los sufragios, seguido por Raúl Pont, de Democracia Socialista, con el 15 por ciento de los votos, y por Valter Pomar, de Articulación de Izquierda, con el 14,65 por ciento de los sufragios (PONT, 2005). El resultado de las elecciones permitió a los representantes de las listas opositoras integrarse a la Dirección Nacional, perdiendo la lista oficialista la mayoría absoluta. La segunda vuelta electoral para elegir presidente del partido contó con la asistencia de 226.324 petistas. Finalmente, el candidato oficial, Ricardo Berzoini, ex Ministro de Trabajo en el período 2004-2005, y diputado federal, fue electo presidente del partido por el 52 por ciento en octubre de 2005.

Analizando los resultados de las elecciones internas del año 2005, de los dieciocho estados donde triunfó la lista oficialista, trece pertenecieron a las regiones del Norte y del Nordeste (Cuadro 1). En ellos se registraron las mayores diferencias en sufragios a favor de la lista oficialista, siendo los estados donde el partido se extendió, siguiendo la estrategia de la Dirección Nacional y ya siendo partido gubernamental (Tabla 1). La expansión en los estados menos politizados y más pobres consolidó el fenómeno denominado "lulismo", manifestando la adhesión al liderazgo gubernamental de Lula da Silva (ZUCCO, 2015).

\footnotetext{
${ }^{3}$ El mandato de José Genoíno como presidente electo del partido se extendía hasta diciembre de 2005.

${ }^{4}$ Entre los acusados se encontraban el presidente del partido José Genoíno y el líder de la bancada parlamentaria, Paulo Rocha.
} 
8 | Rosa María Marcuzzi

Tabla 1 - Elecciones internas del presidente del PT: Porcentaje a favor de la lista vencedora por estado, balotaje celebrado el 9 de octubre de 2005

\begin{tabular}{|c|c|c|c|c|}
\hline & \multicolumn{2}{|c|}{ Lista Oficial Campo Mayoritario } & \multicolumn{2}{|c|}{ Lista opositora Democracia Socialista } \\
\hline & Estados & $\%$ de Diferencia & Estados & \% de Diferencia \\
\hline 1 & Acre $(\mathrm{N})$ & 8 & Bahía (NE) & 8 \\
\hline 2 & Alagoas (NE) & 38 & Espíritu Santo & 44 \\
\hline 3 & Amapá (N) & 54 & Maranhão (NE) & 2 \\
\hline 4 & Amazonas (N) & 50 & Mato Grosso & 16 \\
\hline 5 & Ceará (NE) & 30 & Minas Gerais & 20 \\
\hline 6 & Distrito Federal & 10 & Rio de Janeiro & 12 \\
\hline 7 & Goiás & 19 & $\begin{array}{l}\text { Rio Grande del Norte } \\
(\mathrm{NE})\end{array}$ & 6 \\
\hline 8 & Mato Grosso do Sul & 16 & Rio Grande do Sul & 60 \\
\hline 9 & Paraíba (NE) & 20 & Santa Catarina & 44 \\
\hline 10 & Pará $(\mathrm{N})$ & 40 & & \\
\hline 11 & Paraná & 22 & & \\
\hline 12 & Pernambuco (NE) & 2 & & \\
\hline 13 & Piauí (NE) & 8 & & \\
\hline 14 & Rondônia (N) & 14 & & \\
\hline 15 & Roraima $(\mathrm{N})$ & 22 & & \\
\hline 16 & São Paulo & 34 & & \\
\hline 17 & Sergipe (NE) & 14 & & \\
\hline 18 & Tocantins $(\mathrm{N})$ & 36 & & \\
\hline
\end{tabular}

Fuente: Adaptado de Corsalette (2005).

El PT como partido gubernamental aumentó un 700 por ciento el número de afiliados, la Dirección Nacional partidaria promovió la extensión territorial de la organización y la adhesión al liderazgo gubernamental de afiliados menos politizados. Durante el período estudiado, conservó la adhesión de los movimientos sociales y los programas fueron adoptados con críticas internas. Las elecciones de las autoridades partidarias fueron celebradas con anticipación, triunfando en ellas el candidato de la lista promovida desde el gobierno.

\section{E1 Frente para la Victoria de Argentina: Fragmentación interna, descentralización y activismo político (2003-2007)}

El Frente para la Victoria (en adelante FPV) surgió como fracción política del Partido Justicialista, en ocasión de celebrarse las elecciones presidenciales de abril de 2003. El líder político del FPV y luego presidente electo, Néstor Kirchner, desempeńaba su tercer mandato como gobernador de la Provincia de Santa Cruz, 
perteneciendo a la fracción justicialista provincial Frente para la Victoria Santacruceńa que, bajo la vigencia de la ley de lemas, gobernaba la Provincia de Santa Cruz desde 1991.

En las elecciones generales de abril de 2003, el FPV compitió con otras dos fórmulas presidenciales del Partido Justicialista, avaladas por el Congreso Nacional partidario ${ }^{5}$.

La fragmentación interna del partido no obstaculizó la organización de su base social. Partiendo de una cantidad de afiliados estable desde el inicio de la democracia, cercano a los cuatro millones (MUSTAPIC, 2002), el FPV amplió su base social de tres formas: conservando la base sindical propia del Partido Justicialista, incorporando miembros de los movimientos sociales a la formulación de políticas y a los cargos gubernamentales, y creando organizaciones intrapartidarias.

La base sindical del Justicialismo se conservó, mediante una doble alianza con los gremios dominantes de las dos centrales sindicales, el gremio del transporte perteneciente a la Confederación General del Trabajo - CGT y el gremio de los docentes pertenecientes a la Central de los Trabajadores Argentinos - CTA (ETCHEMENDY, 2013).

Dentro de este universo de organizaciones, la CTA representó el modelo que privilegió el FPV en la ampliación de la base social partidaria. Surgida en oposición al proyecto neoliberal de la década del 90, la CTA reúne a más de doscientas cuarenta organizaciones sindicales y sociales, comprendiendo a trabajadores activos sindicalizados, a desocupados y a jubilados (MIRZA, 2006).

Las coincidencias programáticas vincularon al FPV con otras organizaciones, tales como las Madres de Plaza de Mayo, las Abuelas de Plaza de Mayo, Hijos contra la Impunidad por la Justicia contra el Olvido y el Silencio - HIJOS y el Movimiento de Lesbianas, Gays, Transexuales y Bisexuales - LGTB, entre otras.

A los movimientos sociales extrapartidarios, el FPV sumó la creación del "Movimiento Evita" y de la organización juvenil "La Cámpora" en el primer período de gobierno. El "Movimiento Evita" rescató la forma histórica de movimiento del Justicialismo, definida como superadora de la forma partidaria y el liderazgo de Evita, asociado a la política social. La Cámpora adoptó el nombre de quien fuera uno de los fundadores del Partido Justicialista, diputado nacional y Presidente de la Nación,

\footnotetext{
${ }^{5}$ Las fórmulas estaban integradas por Carlos Menem y Juan Carlos Romero por el "Frente por la Lealtad” y Adolfo Rodríguez Saá y Mechor Posse por el “Movimiento Popular -Unión y Libertad”.
} 
Héctor Cámpora, y reeditó la significación que las organizaciones juveniles habían tenido en el tercer gobierno justicialista. Ambas organizaciones se incorporaron a la organización del Justicialismo a nivel distrital en la Provincia de Buenos Aires.

Las plataformas electorales del FPV, respondieron a los requisitos de la ley de partidos políticos, que exige presentar una plataforma junto a las candidaturas presidenciales. En las elecciones presidenciales de 2003 y 2007, las plataformas electorales del FPV fueron superficiales y con escasas propuestas (D'ALESSANDRO, 2013). Estas plataformas electorales no fueron aprobadas por el Congreso Nacional del Partido Justicialista, tal como lo establece la Carta Orgánica, debido a la situación de acefalía e intervención en que se encontraba el partido.

Desde las elecciones presidenciales del año 1999, las actividades partidarias se encontraban obstaculizadas por la confrontación interna entre el ex-Presidente de la Nación Carlos Menem y el ex-gobernador de la Provincia de Buenos Aires Eduardo Duhalde. Ello derivó en la creación, dos años después, de la Comisión de Acción Política, la que agrupó a catorce gobernadores provinciales. Esta comisión, de carácter transitorio hasta la celebración de las elecciones internas, se constituyó como la máxima autoridad política del partido. Néstor Kirchner formaba parte de la comisión como gobernador de la Provincia de Santa Cruz.

La Comisión de Acción Política remplazó al Consejo Nacional del partido, conformando una estructura que se organizó en torno al predominio interno de los gobernadores y de los dirigentes provinciales ${ }^{6}$.

El predominio de los gobernadores y de los presidentes de los distritos al interior del partido no era excepcional, sino que obedeció a la estructura interna partidaria. Según la Carta Orgánica, las máximas autoridades partidarias nacionales, el Congreso y el Consejo, se integraban por los representantes de los distritos, los gobernadores y los presidentes partidarios del nivel distrital, dejando el dominio del partido a cargo de los representantes distritales (PJ, 2003).

Formalmente los afiliados elegían por el voto directo y secreto los siguientes cargos partidarios: la máxima autoridad ejecutiva del partido, que es el Consejo Nacional, los candidatos partidarios a Presidente y Vicepresidente de la Nación, los miembros de los congresos distritales, de los consejos distritales, de los concejos

\footnotetext{
${ }^{6}$ La Comisión de Acción Política se estableció en la reforma de la Carta Orgánica del Partido del año 2001. Posteriormente el Congreso Nacional Partidario, celebrado el 7 de marzo de 2008, modificó la Carta Orgánica y suprimió la Comisión de Gobernadores como autoridad partidaria.
} 
departamentales y de las unidades básicas. La elección de los representantes distritales al Congreso Nacional lo podían realizar los congresos distritales o los afiliados, siendo establecido por las Cartas Orgánicas de los distritos (PJ, 2003).

Los sistemas electorales utilizados por el partido en el orden nacional fueron el de mayoría con representación de la minoría ( 25 por ciento y siempre que alcancen ese porcentaje de votos válidos emitidos), y primarias abiertas para la elección de los candidatos a Presidente y Vicepresidente de la República (PJ, 2003).

Durante el período bajo estudio no se celebraron elecciones internas de las autoridades partidarias a nivel nacional. La conflictiva situación interna se reflejó en la imposibilidad de elegir a las nuevas autoridades partidarias luego de concluida la presidencia de Carlos Menem, en abril de 2003 (Cuadro 1).

Cuadro 1 - Presidentes del Partido Justicialista en el nivel nacional, período: 2003- 2010

\begin{tabular}{|l|l|l|}
\hline \multicolumn{1}{|c|}{ Presidentes } & \multicolumn{1}{|c|}{ Formas de elección } & \multicolumn{1}{c|}{ Período } \\
\hline Carlos Menem & $\begin{array}{l}\text { Reelecto por el Consejo Nacional partidario } \\
\text { desde 1990. Luego de su detención, desde } \\
\text { junio a noviembre de 2001, reasume como } \\
\text { presidente del partido. }\end{array}$ & $28 / 11 / 2001-27 / 04 / 2003$ \\
\hline Eduardo Fellner & $\begin{array}{l}\text { Asume como presidente interino designado } \\
\text { por la Comisión de Acción Política }\end{array}$ & $11 / 06 / 2003-29 / 03 / 2004$ \\
\hline Acéfalo & Renuncia del presidente interino & $29 / 03 / 2004-06 / 09 / 2005$ \\
\hline Ramón Ruiz & Interventor judicial & $06 / 09 / 2005-23 / 04 / 2008$ \\
\hline Néstor Kirchner & Electo por el Congreso Nacional partidario & $\begin{array}{l}14 / 05 / 2008-29 / 06 / 2009 \\
11 / 11 / 2009-27 / 10 / 2010^{7}\end{array}$ \\
\hline
\end{tabular}

Fuente: Elaboración propia ${ }^{8}$.

El fraccionalismo interno impidió que el nuevo liderazgo gubernamental se impusiera al interior del partido, y los conflictos internos persistieron durante el transcurso del primer gobierno del FPV.

En síntesis, la fragmentación partidaria interna no obstaculizó que el FPV aumentara el activismo, incorporando a nuevos actores sindicales y sociales. La

\footnotetext{
${ }^{7}$ Daniel Scioli fue presidente interino del partido durante cinco meses debido a la presentación de la renuncia del presidente partidario Néstor Kirchner luego de la derrota electoral en las elecciones legislativas celebradas el 28 de junio de 2009, la renuncia no fue aceptada por el congreso partidario.

${ }^{8}$ Entrevista realizada por la autora al Dr. Juan Manuel Abal Medina (ex asesor del Diputado Nacional Néstor Carlos Kirchner, 2009-2010), en su despacho de la Comisión Nacional para la Acreditación y Evaluación Universitaria-CONEAU, Ciudad Autónoma de Buenos Aires, 2 de marzo de 2018.
} 
organización partidaria permaneció acéfala y bajo intervención judicial por la imposibilidad de elegir sus autoridades, lo que impidió la adopción de los programas partidarios y la celebración de las elecciones internas. La situación se normalizaría posteriormente cuando el congreso partidario eligió a Néstor Kirchner como presidente del partido en abril de 2008.

\section{E1 Frente Amplio de Uruguay: activismo politico fragmentado y predominio interno de las fracciones partidarias en el gobierno (2005-2010)}

El Frente Amplio (en adelante FA) surgió como una alianza electoral del Partido Socialista, el Partido Comunista, la Democracia Cristiana, sectores de partidos Colorado y Nacional y otros partidos de izquierda, presentándose en las elecciones de 1971 y alcanzando, en esa oportunidad, el 18 por ciento de los sufragios. Este acontecimiento dio por concluido el bipartidismo de Colorados y Blancos (Partido Nacional) que se habían alternado en el gobierno desde el inicio del siglo XX (GONZÁLEZ, 1990). Desde el inicio de la democracia, los sucesivos referéndums y plebiscitos convocados para decidir una amplia agenda de temas movilizaron a los adherentes del partido. En 1989 el FA participó en el referéndum para derogar la Ley de Caducidad de Pretensión Punitiva del Estado; en el mismo año promovió el voto favorable en el plebiscito de reajuste de las jubilaciones y pensiones; en 1992 participó en el referéndum para derogar la ley de empresas públicas que privatizaba sectores como la telefonía; en 1996 se opuso al plebiscito sobre la reforma constitucional para introducir la elección del presidente por balotaje; y en el año 2003 participó del referéndum para derogar la ley que desmonopolizaba la importación y refinería de petróleo por parte de la empresa estatal Administración Nacional de Combustibles Alcohol y Portland - ANCAP, entre otros (MOREIRA, 2004)? .

Al llegar a las elecciones nacionales de octubre de 2004, el FA contaba con 493.805 adherentes, alcanzando el 20 por ciento del total de electores habilitados en el país (MOREIRA, SELIOS y LIZBONA, 2009). El programa del partido se elaboró sobre la base de la cultura política de izquierda, el que fue modificándose desde una posición anticapitalista, antiimperialista y socialista al progresismo, basado en políticas sociales redistributivas de aumento del gasto social en salud y en

\footnotetext{
${ }^{9}$ El referéndum se aplica para derogar leyes ya votadas, y el plebiscito está destinado a aprobar textos constitucionales. Pueden convocarse junto a las elecciones nacionales o en instancias separadas (MOREIRA, 2004).
} 
educación, negociación colectiva de los salarios y alivio de la deuda externa sin comprometer los recursos públicos destinados a las políticas sociales (MOREIRA, SELIOS y LIZBONA, 2009).

Según el estatuto partidario, los adherentes se organizan en los comités de base, los que designan en asamblea anualmente a la mesa y al secretariado del comité. Los adherentes eligen, cada treinta meses, a dos clases de delegados, aquellos que representan a los comités de bases y a los delegados de las fracciones, llamadas sectores políticos en el Estatuto. Eligen los delegados de los sectores políticos a los plenarios zonales, departamentales y al Plenario Nacional, y a los delegados de las bases a estos organismos y al Congreso Nacional (FA, 2011) ${ }^{10}$.

Los representantes de las fracciones son electos en listas cerradas y bloqueadas en circunscripciones plurinominales para integrar los plenarios zonales, departamentales y el Plenario Nacional y los delegados de los comités de base son electos como candidatos individuales para integrar los plenarios zonales, departamentales, los que a su vez integran el Plenario Nacional y el Congreso Nacional (VERA, 2017).

$\mathrm{El}$ adherente del FA elige representantes a ocho cargos diferentes al interior de la organización, miembros de la mesa política del comité de base al cual pertenece, delegados de los sectores políticos a los plenarios zonal, departamental y nacional y delegados de las bases a los plenarios zonal, departamental, nacional y al Congreso Nacional (FA, 2011).

Siguiendo el Estatuto partidario, la máxima autoridad política permanente, el Plenario Nacional, es un organismo plural integrado por los representantes de las distintas fracciones políticas, por los delegados elegidos por los comités de base y por los parlamentarios e intendentes (FA, 2011). Los delegados de los sectores políticos son elegidos por representación proporcional y se le asegura a cada lista que se presente un delegado en la conformación del Plenario Nacional. Las decisiones se toman por consenso o por mayoría absoluta y se prevé la unanimidad para modificar las bases programáticas y el acuerdo político (FA, 2011).

\footnotetext{
${ }^{10}$ A partir de la reforma estatutaria de 1993, el Plenario Nacional dejó de estar integrado por un número fijo de representantes de los sectores políticos y se conformó a través de elecciones internas de los adherentes (VERA, 2017).
} 
Las elecciones internas para elegir las autoridades partidarias son realizadas cada treinta meses en los ańos en los cuales no se celebran elecciones generales, son elecciones cerradas en las cuales participan solamente los adherentes del partido ${ }^{11}$.

Las renovaciones de las autoridades de los comités de base y de las autoridades partidarias nacionales presentaron irregularidades en el período de desempeño gubernamental del FA. Los comités de base se organizaron durante los períodos electorales, siendo pocos los que funcionaron de forma regular (MOREIRA, SELIOS y LIZBONA, 2009), y las elecciones de las autoridades nacionales excedieron el plazo de treinta meses previsto en el Estatuto ${ }^{12}$.

Desde la asunción de Tabaré Vázquez como Presidente de la República en marzo de 2005, el liderazgo partidario lo asumió el vicepresidente del partido Jorge Brovetto $^{13}$. Ex Rector de la Universidad de la República, Brovetto se incorporó al FA como independiente por invitación de Vázquez. Integró el gabinete presidencial como Ministro de Educación y simultáneamente ocupó la presidencia del partido. Luego fue electo como presidente del partido por el Congreso Nacional en noviembre de 2006, permaneciendo en el cargo hasta junio de 2012.

En el primer período del gobierno nacional del FA, el partido celebró elecciones internas en noviembre de 2006. Esta elección llegó a convocar a 214.040 votantes, superando el número de asistentes de la elección interna del año 2002, cuando alcanzaron los 203.105 votantes (FA, 2006 y 2002).

Las fracciones partidarias predominantes al interior del FA fueron aquellas que simultáneamente ocupaban cargos ministeriales y bancas en el Poder Legislativo: el Partido Socialista (fracción a la que pertenecía el Presidente de la Nación), la Vertiente Artiguista, el Partido Comunista, el Movimiento de Participación Popular, la Asamblea Uruguay y la Alianza Progresista (fracción a la que pertenecía el Vicepresidente de la Nación, Rodolfo Nin Novoa). Las fracciones citadas dominaban

\footnotetext{
${ }^{11}$ En la reforma del Estatuto del año 2011 se incluyó la paridad de género, y la organización interna se complejizó con respecto a la establecida en el Estatuto del año 2002. Se incorporaron a nivel nacional las Coordinadores del Exterior, la Agrupación Nacional de Gobierno y las Comisiones Nacionales, y a nivel subnacional las Regionales del Interior, Agrupaciones de Gobierno Departamentales y Municipales de Gobierno de Montevideo, departamento donde se concentra la mayor cantidad de adherentes (FA, 2011; MOREIRA, SELIOS y LIZBONA, 2009).

${ }^{12}$ Las elecciones internas para las autoridades partidarias se celebraron en los años 1997, 2002, 2006, 2012 y 2016 (VERA, 2017).

${ }^{13}$ Según disposición constitucional, el Presidente de la República no puede participar simultáneamente de organismos directivos de un partido político (art.77, inc.5) (URUGUAY, 1997).
} 
el Plenario Nacional, sumando el 87 por ciento de la representación de las fracciones políticas en el año 2002 y conservando este predominio luego de las elecciones internas de 2006, con el 70 por ciento de los cargos destinados a las fracciones (VERA, 2017).

En síntesis, siendo partido de gobierno, el FA aumentó el número de participantes internos y conservó su programa partidario de cultura de izquierda moderada. Las elecciones internas, de las que participaron un mayor número de adherentes, se celebraron de forma irregular, no cumpliendo los plazos establecidos en el estatuto partidario. Las fracciones que predominaron al interior del partido fueron aquellas que contaban con cargos ministeriales y bancas legislativas, y la presidencia del partido la conservó el candidato oficialista.

\section{El Movimiento Alianza Patria Altiva I Soberana de Ecuador: activismo politico, centralización interna $y$ fusión del liderazgo partidario y gubernamental (2009-2013)}

El movimiento Alianza Patria Altiva I Soberana (en adelante AP) surgió como plataforma electoral de su candidato presidencial Rafael Correa para presentarse en las elecciones generales celebradas en octubre de 2006. Formado unos meses antes de las elecciones, en abril de 2006, adoptó la figura legal de movimiento político. El Tribunal Supremo Electoral los denominaba movimientos políticos independientes, reconocidos por la Constitución de 1998 para participar en los procesos electorales (HIDALGO FLOR, 2002; LACUISSE, 2007) ${ }^{14}$.

Los requisitos para constituir un movimiento político se diferenciaban de la formación de un partido político. Los movimientos requerían para su reconocimiento la presentación de un acta de conformación y del régimen orgánico, la elección de autoridades internas, la obtención de un mínimo porcentaje de adherentes y podían constituirse localmente; por el contrario, el partido político requería incluir un mínimo de afiliados distribuidos territorialmente, la formación de organismos provinciales y sólo se reconocía su actuación en el nivel nacional.

El año 2010, bajo la nueva Constitución y del Código de la Democracia, al reinscribirse como movimiento político, culmina su proceso de formación. Como requisito para su reinscripción, $\mathrm{AP}$ reunió un millón y medio de adhesiones, siendo el movimiento político que más firmas presentó ante el Consejo Nacional Electoral.

\footnotetext{
${ }^{14}$ El Tribunal Supremo Electoral luego de la Reforma Constitucional de 2008 pasará a ser el Consejo Nacional Electoral.
} 
Posteriormente se inició un proceso de afiliación en la capital del país, llegando a sumar 907.003 adherentes ${ }^{15}$.

En el inicio las organizaciones sociales que adhirieron a la candidatura de Rafael Correa para formar AP fueron Jubileo 2000, Iniciativa Ciudadana, Movimiento Alianza Alfarista Bolivariana, Acción Democrática Nacional, Polo Democrático, Foro Urbano, Alternativa Democrática y Ruptura de los 25.

Los programas del partido se elaboraron para las campañas electorales presidenciales de octubre de 2006 y de abril de 2009 ya bajo la nueva Constitución. $\mathrm{La}$ "revolución ciudadana" y el "socialismo del buen vivir" fueron los ejes estructuradores de los programas partidarios, que contaron con la colaboración de los movimientos sociales en su elaboración.

Desde su creación, las máximas autoridades partidarias, el presidente y el vicepresidente de AP, desempeñaron simultáneamente los máximos cargos políticos del gobierno nacional, Rafael Correa la Presidencia de la Nación y Lenin Moreno la Vicepresidencia de la Nación.

Como movimiento político de carácter nacional, cuenta con adherentes permanentes (activistas), con adherentes circunstanciales, quienes firman para cumplir requisitos de participación electoral, y con simpatizantes.

Los adherentes permanentes y los simpatizantes forman parte de los Comités de la Revolución Ciudadana, quedando reservados para los primeros los cargos directivos del movimiento, las candidaturas y la posibilidad de desempeñar cargos remunerados en la organización. Los Comités de la Revolución Ciudadana son los encargados de la formación política y de elegir a los representantes a las diferentes instancias partidarias.

Estos comités fueron organizados a partir de la propuesta gubernamental de conformar Comités de Defensa de la Revolución, enunciada en el discurso de asunción presidencial de Rafael Correa bajo la nueva Constitución, en agosto de 2009. La propuesta del Presidente de la Nación, simultáneamente presidente de AP, fue llamarlos Comités de Vigilancia de la Revolución Ciudadana. Luego, debido a las críticas de los partidarios, de la ciudadanía y de la oposición, se los llamó Comités de la Revolución Ciudadana (HERRERA LLIVE, 2017).

Los comités constituyen la base del partido y eligen a los representantes a las convenciones, en los niveles ascendentes parroquial, distrital, cantonal, provincial y

${ }^{15}$ Información proporcionada por la Coordinación Nacional Técnica de Procesos de Participación Política del Consejo Nacional Electoral de Ecuador, 2018. 
nacional, siendo la Convención Nacional la máxima autoridad del partido. Las convenciones eligen a los candidatos del partido para las elecciones generales según los niveles territoriales y a las autoridades partidarias del respectivo nivel. Las convenciones están integradas por los delegados de los niveles inferiores de su jurisdicción (AP, 2014 y 2009 ${ }^{16}$ ).

Los procesos electorales internos del movimiento político se encuentran bajo el control del Consejo Nacional Electoral ${ }^{17}$.

En el orden nacional, la Convención se reúne por lo menos una vez cada dos años. Integrada por los miembros de la Dirección Nacional, por las autoridades electas hasta el nivel que decida la Dirección Nacional, y por los delegados/as territoriales designados por las direcciones provinciales y circunscripciones especiales del exterior, aprueba el Programa de Gobierno, el Régimen Orgánico del movimiento, elige las autoridades del partido y los candidatos a presidente y vicepresidente (AP, 2014 y $2009^{18}$ ). En la elección de las candidaturas y de las autoridades se aplican los criterios de equidad y de paridad de género. Las direcciones de cada nivel, parroquial, distrital, cantonal, provincial y nacional, son las autoridades políticas permanentes del movimiento. La toma de decisiones en todos los organismos es por consenso o por mayoría simple, constatando el quórum (AP, 2014 y $\left.2009^{19}\right)$.

Durante el primer período completo de gobierno de AP, el que tuvo lugar bajo la nueva Constitución ${ }^{20}$, fueron celebradas dos convenciones, tal como lo establece el estatuto partidario. La primera tuvo su apertura el 18 de septiembre de

16 Régimen Orgánico del Movimiento Alianza Patria Altiva I Soberana. Documento para su reinscripción. 2009. Documento proporcionado por la Coordinación Nacional Técnica de Procesos de Participación Política del Consejo Nacional Electoral de Ecuador, 2018.

17 Artículo 219, inc.4 de la Constitución Nacional y artículo 345 del Código de la Democracia (ECUADOR, 2008).

18 Régimen Orgánico del Movimiento Alianza Patria Altiva I Soberana. Documento para su reinscripción. 2009. Documento proporcionado por la Coordinación Nacional Técnica de Procesos de Participación Política del Consejo Nacional Electoral de Ecuador, 2018.

19 Régimen Orgánico del Movimiento Alianza Patria Altiva I Soberana. Documento para su reinscripción. 2009. Documento proporcionado por la Coordinación Nacional Técnica de Procesos de Participación Política del Consejo Nacional Electoral de Ecuador, 2018.

${ }^{20}$ La fórmula del movimiento AP triunfó en el balotaje presidencial de noviembre de 2006 y accedió al gobierno en enero de 2007. Sancionada la nueva Constitución en septiembre de 2008, se convocaron a elecciones celebradas en abril de 2009, triunfando la fórmula de AP que gobernó desde agosto de 2009 hasta mayo de 2013. 
2010 en la ciudad de Montecristi en la Provincia de Manabí y se clausuró dos meses después, en la ciudad de Guayaquil, capital de la Provincia de Guayas. La segunda Convención Nacional tuvo lugar el 10 de noviembre de 2012 en la ciudad de Quito, capital de la Provincia de Pichincha y capital del país. Las convenciones estuvieron presididas por Rafael Correa, quien, a su vez, desempeńaba la Presidencia de la Nación.

En síntesis, en el primer período de gobierno, el movimiento AP aumentó el número de adhesiones para inscribirse como movimiento político, formación en la que habían confluido inicialmente diversas organizaciones sociales y los programas partidarios manifestaron las posteriores orientaciones del gobierno. En la celebración de las convenciones nacionales, se centralizaron la designación de los candidatos y la aprobación de los programas partidarios, fusionando la presidencia del partido con la Presidencia de la Nación.

\section{Análisis comparado de los partidos gubernamentales: activismo politico, centralización interna y subordinación al liderazgo gubernamental}

Los cuatro casos seleccionados el PT, el FPV, el FA y AP aumentaron el activismo político interno y se vincularon con los movimientos sociales. Las irregularidades en la celebración de las elecciones internas y el predominio de los candidatos oficialistas son los atributos que señalan el proceso de centralización interna y de pérdida de autonomía partidaria frente al gobierno.

El PT incrementó el activismo interno de sus afiliados. El adelantamiento de las elecciones de las autoridades partidarias debido a los casos de corrupción y el triunfo del candidato oficialista, amplio en los estados de expansión reciente del partido y escenarios del fenómeno llamado "lulismo", verifica la centralización interna y la subordinación partidaria al liderazgo gubernamental

El FA incrementó la adhesión al interior del partido. Las irregularidades se presentaron en la celebración de las elecciones internas, no cumpliendo con los plazos establecidos y conservando la presidencia partidaria a cargo del candidato impulsado por el liderazgo gubernamental.

El FPV prescindió de la organización partidaria en su primer período de gobierno. Ello no fue obstáculo para que se incrementara el activismo político de sus adherentes, incorporando, por coincidencias programáticas, a diversos movimientos sociales. La acefalía y la intervención del partido por la autoridad electoral fueron 
superadas posteriormente con la elección de Néstor Kirchner como presidente del partido.

El movimiento AP presentó creciente activismo político interno desde su organización como movimiento social. Las convenciones nacionales partidarias, al proclamar los candidatos bajo la dirección de la presidencia partidaria, simultáneamente ejerciendo la Presidencia de la Nación, fusionaron el liderazgo gubernamental y el liderazgo partidario.

En síntesis, los cuatro partidos analizados, al acceder al gobierno, presentaron un creciente activismo político combinado con procesos de centralización interna y de subordinación de la organización partidaria al liderazgo gubernamental, lo que contradice lo afirmado por el enfoque del politólogo Kaare Strom sobre la presencia del activismo político en organizaciones partidarias descentralizadas y autónomas del liderazgo gubernamental.

\section{Conclusiones}

Los partidos políticos son actores sociales y estatales simultáneamente, y ello los distingue de las demás organizaciones sociales. Esta simultaneidad de desempeños en la sociedad y en el Estado condiciona a la organización interna partidaria.

Del presente estudio se concluye que los partidos, al acceder al gobierno, incrementaron el activismo político interno de forma simultánea al proceso de centralización y de subordinación al liderazgo gubernamental. Estas particularidades se presentaron en los partidos gubernamentales latinoamericanos, siendo actores relevantes de los sistemas políticos.

Del análisis empírico realizado se concluye que los partidos políticos gubernamentales estudiados cuentan con bases sociales organizadas en multiplicidad de movimientos sociales, lo que indica sociedades civiles fuertes donde transcurren los procesos democráticos de socialización política. Estos partidos políticos han incorporado a sus estructuras, de manera formal o informal, a los movimientos sociales, contribuyendo así a politizar las agendas de los poderes estatales y condicionando la influencia de expertos y de elites económicas.

El proceso de creciente activismo político partidario no ha sido menguado por la progresiva centralización interna en la dinámica de las organizaciones, reflejada en la alteración de sus procedimientos intrapartidarios y en la pérdida de autonomía frente al liderazgo gubernamental, lo que contradice el enfoque teórico institucional sobre el diseño descentralizado propio de los partidos con activismo interno. 
Resta a futuras investigaciones profundizar las particularidades de los partidos políticos latinoamericanos y la relación del activismo político con las transformaciones en el ejercicio de gobierno.

Rosa María Marcuzzi é Doutora em Ciência Política, pela Escuela de Política y Gobierno da Universidad Nacional de San Martín (UNSAM). Professora-investigadora da Universidad Católica Argentina - UCA. E-mail: rosamariamarcuzzi@uca.edu.ar.

\section{Referências}

AMARAL, Oswaldo. O que sabemos sobre a organização dos partidos políticos: uma avaliação de 100 anos de literatura. Revista Debates, v. 7, p. 11-32, mai./ago. 2013.

BRECKENRIDGE-JACKSON, Ian et al. Los Movimientos Sociales Latinoamericanos y el Proceso del Foro Social Mundial. In: ALMEIDA, Paul; CORDERO ULATE, Allen (Eds.). Movimientos sociales en América Latina: perspectivas, tendencias y casos. Buenos Aires: CLACSO, 2017. p. 160180.

CORSALETTE, Conrado. Berzoini vence e quer pacto para reeleger Lula. Folha do São Paulo, 12 out. $2005 . \quad$ Disponible en: <https://acervo.folha.com.br/leitor.do? numero=16592\&anchor $=5247781$ \&origem $=$ busca\&originU $\mathrm{RL}=\& \mathrm{pd}=\mathrm{b} 2 \mathrm{f} 21 \mathrm{e} 33 \mathrm{bbb7ebe} 51 \mathrm{a} 079 \mathrm{be} 2316 \mathrm{e} 26 \mathrm{a} 2>$. Acceso: 20 nov. 2020.

D'ALESSANDRO, Martín. Las Plataformas Electorales en la Argentina Moderna. América Latina Hoy, v. 65, p. 107-139, dic. 2013.

ECUADOR. Constitución de la República del Ecuador. Ciudad Alfaro, Montecristi, Provincia de Manabí, 2008.

ETCHEMENDY, Sebastián. La "doble alianza" gobierno- sindicatos en el kirchnerismo (20032012). Orígenes, evidencia y perspectivas. In: ACUÑA, Carlos (Comp.). ¿Cuánto importan las instituciones? Gobierno, Estado y actores en la política argentina. Buenos Aires: SXXI editores; Fundación OSDE, 2013. p. 291- 324.

FRENTE AMPLIO (FA). Elecciones internas, 26 de mayo de 2002. Secretaría de la Comisión Nacional de Organización, Montevideo.

FRENTE AMPLIO (FA). Elecciones internas, 12 de noviembre de 2006. Secretaría de la Comisión Nacional de Organización, Montevideo.

FRENTE AMPLIO (FA). Estatuto, contiene modificaciones del Plenario Nacional de diciembre de 2011. 2011. Disponible en: <http://www.frenteamplio.org.uy/index.php?Q=articulo\&ID=1012>. Acceso: 5 oct. 2018. 
GONZÁLEZ, Luis. Legislación electoral y sistemas de partidos: el caso Uruguayo. Revista Uruguaya de Ciencia Política, n. 4, p. 9-27, ene./dic. 1990.

HERERA LLIVE, Klever Vinicio. Las organizaciones de base de Alianza PAIS: el papel de los comités de la revolución ciudadana en la movilización política. Análisis Político, n. 91, p. 96-109, sep. 2017.

HIDALGO FLOR, Francisco. Elecciones en Ecuador: Quiebra de los Partidos Políticos y Presencia del Movimiento Indígena. Ciencias Sociales, v. 98, p. 87-97, 2002.

LACUISSE, Marie Esther. Los movimientos políticos locales en el escenario electoral. Iconos. Revista de Ciencias Sociales, n. 27, p. 37-45, ene./abr. 2007.

LLAMAZARES VALDUVIECO, Iván. El análisis comparado de los fenómenos políticos. 1996. Revista Mexicana de Sociología, v. 58, p. 153-169, jan./mar. 1996.

MARINI, Ruy. El movimiento obrero brasileño. Cuadernos Políticos, n. 46, p. 5-23, abr./jun. 1986.

MIRZA, Christian. Movimientos sociales y sistemas políticos en América Latina: la construcción de nuevas democracias. Buenos Aires: CLACSO, 2006. (Programa Regional de Becas CLACSO).

MOREIRA, Constanza. Resistencia Política y Ciudadanía: Plebiscitos y Referéndums en el Uruguay de los 90. América Latina Hoy, v. 36, p. 17-45, abr. 2004.

MOREIRA, Constanza; SELIOS, Lucia; LIZBONA, Alexandra. La renovación programática del Frente Amplio: una mirada al proceso reciente (2004-2009). Uruguay: FES- ICP, 2009.

MOVIMIENTO ALIANZA PATRIA ALTIVA I SOBERANA (AP). La Convención Nacional del Movimiento Alianza País, Patria Altiva I Soberana. Esmeraldas, 1 mayo 2014.

Disponible en: <https://reformaspoliticas.org/wp-content/uploads/2020/05/Estatuto-AlianzaPa\%C3\%ADs.pdf>. Acceso: 15 nov. 2020.

MUSTAPIC, Ana María. Del Partido Peronista al Partido Justicialista. Las Transformaciones de un Partido Carismático. In: CAVAROZZI, Marcelo; ABAL MEDINA, Juan Manuel (Comps.). El asedio a la política. Los partidos latinoamericanos en la era neoliberal. Rosario: Homo Sapiens Ediciones, 2002. p. 137- 161.

PARTIDO DE LOS TRABAJADORES (PT). Estatuto. Aprobado en reunión del Directorio Nacional, 5 de octubre de 2007. [S.1], 2007. Disponible en: <https://www.justicaeleitoral.jus.br/arquivos/estatuto-do-partido-de-5-10-2007-resolucao-tseno.2008/rybena_pdf?file=https://www.justicaeleitoral.jus.br/arquivos/estatuto-do-partido-de-5-102007-resolucao-tse-no.2008/at_download/file>. Acceso: 30 oct. 2020.

PARTIDO DE LOS TRABAJADORES (PT). Estatuto. Aprobado en reunión del Directorio Nacional, 11 de marzo de 2001. [S.1.], 2001. Disponible en: <https://www.justicaeleitoral.jus.br/arquivos/estatuto-do-partido-de-11-3-2001-resolucao-tseno.2002/rybena_pdf?file=https://www.justicaeleitoral.jus.br/arquivos/estatuto-do-partido-de-11-32001-resolucao-tse-no.2002/at_download/file>. Acceso: 30 oct. 2020.

PARTIDO JUSTICIALISTA (PJ). Carta Orgánica. Aprobada según las modificaciones introducidas por el Congreso Nacional Partidario realizado el 20 de Septiembre de 1991, el 17 de Julio de 1998, el 10 de noviembre de 2001, el 5 de Noviembre de 2002 y el 24 de Enero de 2003. [S.1.], 2003. Disponible en: <http://americo.usal.es/oir/opal/Documentos/Argentina/Partido\%20Justicialista/CartaOrganicaPJ.p df>. Acceso: 5 nov. 2020. 
PEREIRA DA SILVA, Fabricio. Vitórias na crise. Trajetórias das esquerdas latino-americanas contemporâneas. Rio de Janeiro: Ponteio, 2011.

PONT ultrapassa Pomar em disputa por vaga no 2o turno. Folha de S. Paulo, 25 set. 2005. Disponible en: https://www1.folha.uol.com.br/fsp/brasil/fc2509200515.htm.

Fecha de consulta: 2 diciembre de 2020.

SECCO, Lincoln. História do PT. Cotia, SP: Ateliê Editorial, 2018.

STROM, Kaare. A Behavorial Theory of Competitive Political Parties. American Journal of Political Science, v. 34, n. 2, p. 565-598, may 1990.

URUGUAY. Constitución de la República Oriental del Uruguay. Montevideo, 1997.

VAN DYCK, Brandon. Why Party Organization Still Matters: The Workers' Party in Northeastern Brazil. Latin American Politics and Society, v. 56, n. 2, p. 1-26, 2014.

VERA, Bruno. Esquerdismo na América Latina (2002- 2008): o racha à esquerda no "Frente Amplio" em perspectiva comparada com o racha á esquerda no Partido dos Trabalhadores. 2017. 226 f. Dissertação (Mestrado em Ciência Política) - Programa de Pós-graduação em Ciência Política, Universidade de São Paulo (USP), São Paulo, 2017.

ZUCCO, César. Estabilidad sin raíces: Institucionalización del sistema de partidos en Brasil. In: TORCAL, Mariano (Comp.). Sistemas de partidos en América Latina. Causas y consecuencias de su equilibrio inestable. Barcelona: Anthropos Editorial, 2015. p. 78-107.

Texto recebido em 07 de agosto de 2020. Aprovado em 23 de novembro de 2020. 